\title{
Evaluación del establecimiento de la métrica de enrutamiento WCETT en una red de radio cognitiva
}

\section{WCETT routing-metric setup assessment on a cognitive radio net- work}

\section{Édgar Alirio Aguirre}

Ingeniero en Control, estudiante de la maestría en Ciencias de la información y la Comunicaciones. Docente de la Corporación Universitaria Minuto de Dios. Bogotá, Colombia.

Contacto:eaaguirreb@correo.udistrital.edu.co

\section{Luis F. Pedraza}

Ingeniero Electrónico, magíster en Ciencias de la Información y las Comunicaciones, estudiante de doctorado en Ingeniería de Sistemas y Computación de la Universidad Nacional de Colombia. Docente e investigador de la Universidad Distrital Francisco José de Caldas. Bogotá, Colombia.

Contacto:lfpedrazam@udistrital.edu.co

\section{Gabriel A. Puerta A.}

Licenciado en Electrónica, estudiante de la Maestría en Ciencias de la Información y las Comunicaciones de la Universidad Distrital Francisco José de Caldas. Bogotá, Colombia.

Contacto:gapuertaa@correo.udistrital.edu.co

Fecha de recepción: 26 de marzo de 2012

Clasificación del artículo: Investigación

Fecha de aceptación: 27 de noviembre de 2012

Financiamiento: Universidad Distrital

Palabras clave: CRCN, NS2, radio cognitiva, WCETT.

Key words: CRCN, NS2, cognitive radio, WCETT.

\section{RESUMEN}

Este artículo presenta la evaluación del establecimiento de la métrica de enrutamiento WCETT (Weighted Cumulative Expected Transmission
Time) para una red de radio cognitiva basado en la aplicación CRCN (Cognitive Radio Cognitive Network Simulator) la cual se desarrolla bajo el entorno de simulación NS2 (Network Simulator 2) donde se comparó el throughput de los proto- 
colos MAC802.11, MACCON, MACN, Simple y TDMA.

\section{ABSTRACT}

This paper presents an evaluation of the WCETT (Weighted Cumulative Expected Transmission Time) routing metric setup for a cognitive radio network. This metric setup is based on the software application called CRCN (Cognitive Radio Cognitive Network Simulator), which runs over the simulation environment called NS2 (Network Simulator 2). Comparative experiments to assess throughput values were conducted for the following protocols: MAC802.11, MACCON, MACN, Simple and TDMA.

\section{INTRODUCCIÓN}

El mundo cambiante de las redes de comunicaciones modernas busca adaptarse a las nuevas necesidades de los usuarios pero, además, busca nuevos métodos para utilizar los recursos de forma más eficiente, recursos como la energía, el ancho de banda, la velocidad de la información y el espectro electromagnético. Bajo la visión de optimizar políticas de asignación del espectro, se puede definir radio cognitiva como un sistema de comunicaciones inalámbrico e inteligente con la capacidad de adaptarse al entorno que le rodea y modificar sus parámetros en función de la señal de radiofrecuencia con la que esté trabajando [1], permitiendo al usuario compartir y usar el espectro de una manera oportunista. Esto conlleva a realizar el acceso al espectro de forma dinámica, teniendo dos tipos de usuarios: los primarios (licenciados) y los secundarios (no licenciados); los primeros, pagan por usar el espectro y poseen mejores características de calidad de servicio; los segundos, no pagan por acceder a usar el espectro pero deben esperar a encontrar espacios libres para poder en-rutar tráfico sin afectar e interferir a los usuarios primarios. Por lo tanto, la radio cognitiva se convierte en un juego de ajedrez [2], en búsqueda de huecos del espectro para cambiar la información. Esto requiere capacidad cognitiva [1] y capturar información del entorno de radio [3], asimismo, debe ser reconfigurable para lograr ser dinámico en el sensado y así redireccionar la información. Esto debe estar fundamentado por la tecnología y el hardware necesario que soporte esta misión [4].

Esta investigación se basa en la evaluación de la métrica de enrutamiento WCETT con diferentes protocolos Mac por medio de la aplicación $C R C N$ bajo el entorno de simulación NS2, esta métrica plantea la evaluación de todos los posibles caminos de enrutamiento desde rutas entre nodos y diferentes canales del espectro, esta es la mayor ventaja de la propuesta de radio cognitiva para poder tener la capacidad de reutilizar el espectro y así usar los canales y frecuencias que no están siendo utilizados, la contribución de este artículo se basa en comparar el throughput resultante de la simulación como aporte a la evaluación de la métrica. Analizando los tiempos de establecimiento con WCETT y evidenciando la necesidad de crear nuevas métricas de enrutamiento para radio cognitiva.

En la segunda parte de este artículo se describen los elementos de la capa de control de acceso al medio (MAC) que se usaron en la simulación, la métrica de enrutamiento y los protocolos, en la tercer parte se explica el desarrollo del experimento y el escenario de movilidad, en la cuarta parte se describen los resultados, en la quinta se realiza la discusión y en la última se enuncian las conclusiones. 


\section{investigación}

\section{PROTOCOLOS CAPA MAC}

En las simulaciones realizadas se trabajó con cinco protocolos por medio de la métrica WCETT, a continuación se describen cada uno de ellos.

\subsection{Métrica WCETT}

Inicialmente, el estudio de la métrica WCETT se centra en nodos fijos de redes locales, donde el objetivo es seleccionar un camino de un alto rendimiento para enviar información entre el origen y el destino [5].

La métrica asigna pesos a los vínculos individuales, los cuales se llaman Expected Transmission Time (ETT), esta es una función de la tasa de perdida y el ancho de banda del enlace, la métrica de interés es Weighted Cumulative ETT (WCETT), figura 1, este es un valor que aumenta dependiendo del número de enlaces que tenga la red y ETT es una estimación del retardo de extremo a extremo, que experimenta un paquete que viaja por un camino entre dos nodos X Y en un enlace $i$.

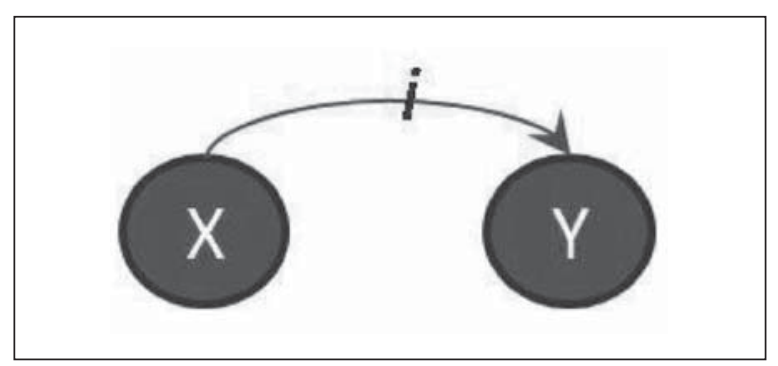

Figura 1. Topología WCETT

Fuente: elaboración propia.

Para calcular WCETT existen varias expresiones, pero la que mejor describe el método se resume en la ecuación (1).

$$
\text { WCETT }=(1-\beta) * \sum_{i=1}^{n} E_{T}+\beta * \max _{1<j<k} X_{j}
$$

Donde $\beta$ es un parámetro autoajustable y debe estar entre $0 \leq \beta \leq 1$, esta ecuación es un equilibrio entre el bien global y el egoísmo, lo que representa un nivel de cooperación entre los nodos de la red. El primer término es la suma de tiempos de transmisión a lo largo de todos los saltos en la red, esto refleja el consumo total de recursos a lo largo de este camino [5]; el segundo término refleja el conjunto de saltos que tendrá el mayor impacto en el rendimiento de este camino. Este promedio ponderado puede ser visto como un intento de equilibrar los dos términos. De todos los caminos se selecciona el mejor WCETT y por esta ruta se envía la información.

\subsection{2 .11}

El estándar 802.11 define el uso de los dos niveles inferiores del protocolo OSI, desde Wi-Fi para redes wireless de área local. En la práctica, Wi-Fi admite ordenadores portátiles, equipos de escritorio, asistentes digitales personales (PDA) o cualquier otro tipo de dispositivo de alta velocidad con propiedades de conexión también de alta velocidad (11 Mbps o superior) dentro de un radio de varias decenas de metros en ambientes cerrados (de 20 a 50 metros en general) [6], o dentro de un radio de cientos de metros al aire libre, el estándar 802.11 establece los niveles inferiores del modelo OSI para las conexiones inalámbricas, por ejemplo:

- La capa física (PHY) ofrece tres tipos de codificación de información.

- La capa de enlace de datos compuesta por dos subcapas: control de enlace lógico (LLC) y control de acceso al medio (MAC)

\subsection{TDMA}

Acceso múltiple por división de tiempo (TDMA) es la tecnología digital de transmisión que permite que un número de usuarios tengan acceso a un solo canal de radiofrecuencia sin interferencia, 
asignando ranuras de tiempo únicas a cada usuario dentro de cada canal. El esquema digital de la transmisión de TDMA multiplexa varias señales sobre un solo canal [7].

\subsection{Simple}

Mac-simple es un protocolo propio de NS2 que se usa para pruebas, está construido bajo el protocolo MAC y tiene un campo para eventos en la traza de tráfico, también tiene un tiempo de espera, un tiempo de envío y recepción, pero en su esencia se basa en el protocolo MAC.

\subsection{MACCON}

Este protocolo es propio de CRCN, siendo un protocolo multi-canal y se basa en el protocolo MAC, este selecciona al azar un canal de un conjunto predefinido de canales, tomando la decisión de selección de canal en la capa MAC [8].

\subsection{MACNG}

El protocolo MACNG es propio de CRCN y su operación se divide en dos fases. En la primera fase, cada nodo envía paquetes de estrategia con el canal preferido de recepción. Si hay un canal disponible, entonces se selecciona como el canal preferido de recepción. Si no hay un canal disponible, las acciones del canal se realizan con el nodo que se encuentra más lejos de este. En la segunda fase, el nodo utilizará el canal seleccionado en la primera fase para enviar y recibir datos. Los algoritmos de selección de canal que se usan están construidos bajo reglas simples, que evitan la colisión [9].

\section{METODOLOGÍA}

El funcionamiento de la aplicación CRCN de radio cognitiva de la métrica WCETT se planteó
Tabla 1. Características del entorno de simulación

\begin{tabular}{|c|c|}
\hline \\
\hline \multicolumn{2}{|l|}{$\begin{array}{l}\text { Escenario de simulación } \\
\text { Numero de nodos }\end{array}$} \\
\hline \multicolumn{2}{|l|}{ Numero de nodos } \\
\hline Phy & WirelessPhy \\
\hline Antena & Omnidireccional \\
\hline $\begin{array}{l}\text { Protocolo de enruta- } \\
\text { miento (métrica) }\end{array}$ & WCETT \\
\hline Tiempos simulación & 50 segundos \\
\hline Interfaces & 4 \\
\hline Canales & 9 \\
\hline Mac simulada & $\begin{array}{l}\text { 802.11-MACCON-MACNG- } \\
\text { Simple-TDMA }\end{array}$ \\
\hline
\end{tabular}

Fuente: elaboración propia.

con las características que se describen en la tabla $1 \mathrm{y}$ con el escenario que se presenta en la figura 2 .

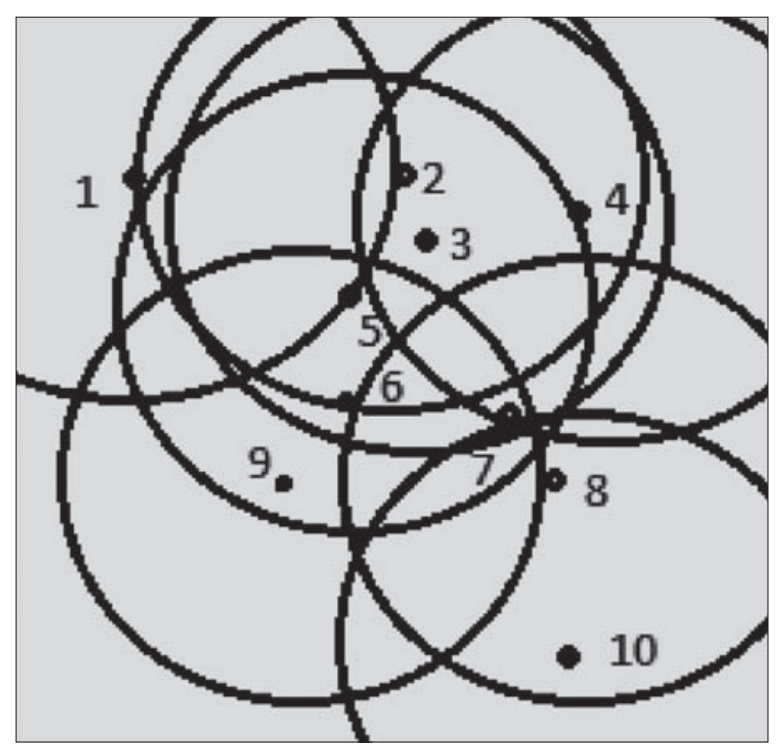

Figura 2. Escenario de movilidad con 10 nodos Fuente: elaboración propia.

El análisis del archivo de tráfico entregado por NS2 presenta el establecimiento de los protocolos, donde la métrica WCETT establece la evaluación de las mejores rutas, por lo tanto, es importante aclarar que el tráfico generado solo es de 
establecimiento y esto es importante para el análisis de las gráficas, entre más throughput presente el protocolo, indica que es necesaria más información para el establecimiento; por el contrario, entre menos throughput exista, el protocolo genera menos información para el establecimiento de las rutas.

\section{RESULTADOS}

Uno de los elementos más importantes en el análisis de las redes de comunicaciones es el parámetro del throughput, que permite determinar la capacidad de la red [10], [11]. En la figura 3 se presenta la comparación del throughput de los protocolos evaluados con la métrica $W C E T T$ de enrutamiento.

El archivo de tráfico generado por NS2 presenta los eventos de la simulación. Para este caso particular, un mayor porcentaje de eventos se refieren al establecimiento de los protocolos. En la tabla 2 se presenta la relación de eventos por protocolo, esto quiere decir, la cantidad de eventos para realizar actividades de envío de información, recepción, paquetes descartados y retransmisiones.

El escenario de simulación se planteó con 10 nodos, en la tabla 3 se observa el throughput para los nodos con los protocolos descritos.

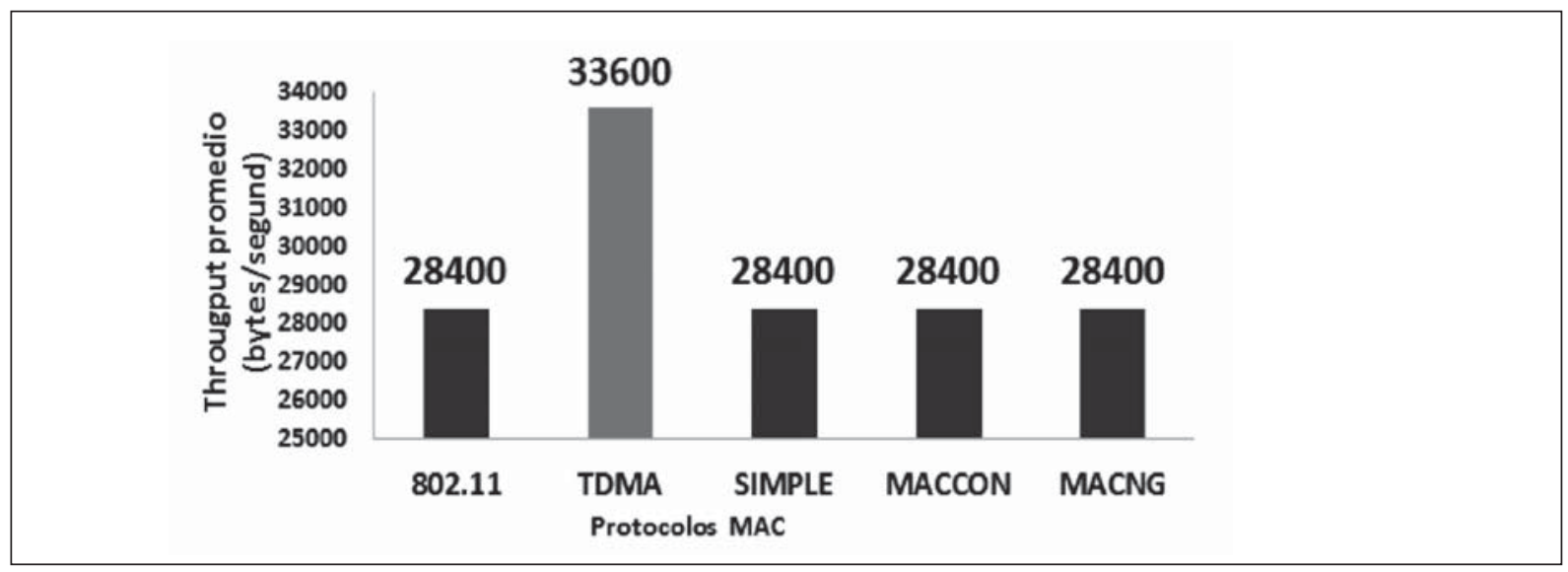

Figura 3. Throughput promedio de protocolos MAC usando la métrica WCETT Fuente: elaboración propia.

Tabla 2. Numero de eventos para cada protocolo MAC

\begin{tabular}{|c|c|c|c|c|c|}
\hline & Enviados & Descartados & Recibidos & Retransmisiones & Total de eventos \\
\hline 802.11 & 512 & 102 & 282 & 0 & 896 \\
\hline MACCON & 3622 & 923 & 561 & 2 & 5108 \\
\hline MACNG & 4572 & 634 & 252 & 0 & 5458 \\
\hline Simple & 4588 & 1174 & 546 & 2 & 6310 \\
\hline TDMA & 11871 & 1603 & 14137 & 0 & 27611 \\
\hline
\end{tabular}

Fuente: elaboración propia. 


\section{investigación}

Tabla 3. Throughput para cada nodo por protocolo

\begin{tabular}{|l|l|l|l|l|l|}
\cline { 2 - 6 } \multicolumn{1}{c|}{} & 802.11 & MACCON & MACNG & Simple & TDMA \\
\hline Nodo 0 & 28400 & 28400 & 28400 & 28400 & 33600 \\
\hline Nodo 1 & 28400 & 28400 & 28400 & 28400 & 33600 \\
\hline Nodo 2 & 28400 & 28400 & 28400 & 28400 & 33600 \\
\hline Nodo 3 & 28400 & 28400 & 28400 & 28400 & 33600 \\
\hline Nodo 4 & 28400 & 28400 & 28400 & 28400 & 33600 \\
\hline Nodo 5 & 33600 & 28400 & 28400 & 28400 & 33600 \\
\hline Nodo 6 & 28400 & 28400 & 28400 & 28400 & 33600 \\
\hline Nodo 7 & 28400 & 28400 & 28400 & 28400 & 33600 \\
\hline Nodo 8 & 28400 & 28400 & 28400 & 28400 & 33600 \\
\hline Nodo 9 & 28400 & 28400 & 28400 & 28400 & 33600 \\
\hline
\end{tabular}

Fuente: elaboración propia.

\section{DISCUSIÓN}

La aplicación CRCN contiene un modelo de movilidad predefinido, el cual es aleatorio y se enlaza con otro modelo de topología llamado topo4, el cual establece la posición inicial de los nodos, estos modelos de topologías y movilidad pretenden simular el comportamiento de usuarios en un entorno, desafortunadamente no son muy reales, la aplicación permite seleccionar otros modelos de movilidad de NS2 o también crear modelos para probarlos, algunos normalmente usados son "random way point", "gauss markov", "GM", "rpgm" entre muchos otros [9].

Todos los eventos de la simulación son de establecimiento, por lo que realmente no se genera un tráfico real de información, en la figura 3 se evidencia que el mayor throughput lo tiene el protocolo TDMA, como se mencionó, esto no es lo mejor debido a que el tráfico generado es de establecimiento y no es para transporte de información, desde este análisis el resto de protocolos se comportan mejor que TDMA porque necesitan menos información para realizar el es- tablecimiento. Para los protocolos 802.11, Simple, MACCON y MACNG el throughput fue de 28400 bytes/segundo a diferencia de TDMA que presento 33600 bytes/segundo.

En la tabla 2 se observa la distribución de los eventos de tráfico por protocolo simulado, donde 802.11 uso menos eventos para realizar el establecimiento de la conexión, esto se refleja en los tiempos y en la mejora del rendimiento de la red y la optimización del uso del ancho de banda. El caso contrario es el del protocolo TDMA que usa un mayor número eventos.

\section{CONCLUSIONES}

Según la aplicación de simulación CRCN, el protocolo MAC que mejor throughput presenta es TDMA, pero el análisis de los archivos de traza de tráfico indica que la información existente es sólo de establecimiento, por lo tanto, TDMA no es el protocolo que mejor se comporta; al contrario, es el que más tiempo de establecimiento necesita. Por lo tanto, 802.11 es el que presenta mejor ren- 


\section{investigación}

dimiento en el establecimiento de la conexión. El software de simulación NS2 trabaja bajo eventos discretos, de aquí se observa que TDMA necesita más eventos para el establecimiento, lo contrario se observa para 802.11 .

La aplicación CRCN plantea el enrutamiento con la selección de la métrica WCETT, aunque presenta limitaciones como la falta de datos de los valores de las rutas con el valor de WCETT para verificar la eficacia de la métrica. Así mismo, se encontró que los modelos de movilidad ya tienen las rutas preestablecidas, por lo que estos modelos no son dinámicos, son estáticos.
Finalmente, se puede afirmar que radio cognitiva promete convertir las comunicaciones inalámbricas en redes más inteligentes, útiles y eficientes, consiguiendo un mejor uso del espectro, así mismo, se observa la importancia del trabajo en protocolos MAC para optimizar los tiempos de establecimiento. Como trabajo futuro se propone el desarrollo de nuevas métricas de enrutamiento, que sean capaces de determinar las características de una red dinámica como lo es la radio cognitiva, para así seleccionar las mejores rutas por medio de métodos de inteligencia artificial que permitan un trabajo cooperativo entre los nodos de comunicación.

\section{Referencias}

[1] S. Haykin, "Cognitive radio: brain-empowered wireless communications", IEEE Journal on Selected Areas in Communications, Vol. 23, pp. 201-220, 2005.

[2] J. Mitola, III and G. Q. Maguire, Jr., "Cognitive radio: making software radios more personal", Personal Communications, IEEE, Vol. 6, pp. 13-18, 1999.

[3] F. K. Jondral, "Software-defined radiobasic and evolution to cognitive radio", in EURASIP Journal on Wireless Communication and Networking, pp. 275-283, 2005.

[4] R. Draves, J. Padhye and B. Zill, "Routing in multi-radio, multi-hop wireless mesh networks", Proceedings of the 10th annual international conference on Mobile computing and networking, Philadelphia, PA, USA, 2004

[5] Z. Wei, Z. Dongbo and Q. Daji, "Comparative study of routing metrics for multiradio multi-channel wireless networks", in Wireless Communications and Networking
Conference, 2006. WCNC 2006. IEEE, 2006, pp. 270-275.

[6] W. World, (2011), Descripción General IEEE 802.11 Redes locales inalámbricas. Available: http://wifiw.com/294/descripcion-general-ieee-802-11-redes-localesinalambricas.html

[7] T. Sekimoto and J. Puente, "A Satellite Time-Division Multiple-Access Experiment", Communication Technology, IEEE Transactions on, Vol. 16, pp. 581-588, 1968.

[8] M. Husain, A. Carneiro, H. Khalife and S. Fdida, "SURF: A Distributed Channel Selection Strategy for Data Dissemination in Multi-Hop Cognitive Radio Networks", 2011.

[9] J. Zhong and J. Li, (2010), Cognitive Radio Cognitive Network Simulator. Available: http://stuweb.ee.mtu.edu/ ljialian/index. htm 
[10] M. A. Alzate, N. M. Pena and M. A. Labrador, "Capacity, bandwidth and available bandwidth concepts for wireless ad hoc networks", in Military Communications Conference, 2008. MILCOM 2008. IEEE, 2008, pp. 1-7.
[11] M. A. Alzate, J. C. Pagan, N. M. Pena and M. A. Labrador, "End-to-end bandwidth and available bandwidth estimation in multi-hop IEEE $802.11 \mathrm{~b}$ ad hoc networks", in Information Sciences and Systems, 2008. CISS 2008. 42nd Annual Conference on, 2008, pp. 659-664. 